Results 12 months after initiation of the new integrated service, the proportion of patients with GC and RCC reviewed by PC at diagnosis of incurable disease had increased from $26 \%$ and $16 \%$ to $80 \%$ and $93 \%$ respectively. $79 \%$ (RCC) and $72 \%$ (GC) had severe or overwhelming psychosocial needs. $18 \%$ (RCC) and 25\% (GC) had severe or overwhelming physical needs.

47 patients had died at time of analysis (16 RCC and 31 GC). Median time from review to death was 134 days (range 20-318 days). This compares to a median time between PC referral and death in the baseline cohort of 98 days (GC) and 83.5 days (RCC).

Conclusions The Integrated service proactively identifies patients with PC needs earlier and has highlighted the high burden of psychosocial needs. This study will underpin service development and improvement to include proactive intervention.

\section{IMPROVING THE PRESCRIBING IN A GENERAL HOSPITAL OF 'AS REQUIRED' ANTICIPATORY MEDICATIONS FOR END OF LIFE SYMPTOMS, FOLLOWING THE INTRODUCTION OF AN ELECTRONIC PRESCRIBING 'ORDER-SET'}

Debra Swann, Louise Renton, Caroline Adams, Elizabeth Heitz, Devesh Patel. Croydon University Hospital

\subsection{6/bmjspcare-2018-ASPabstracts.159}

Background Availability of anticipatory medications for common end of life (EOL) symptoms is a quality indicator measured in recent hospital care of the dying audits. Local results from the 2013 audit demonstrated poor uptake of anticipatory prescribing. In 2014, the Trust moved to electronic patient records and prescribing across the acute trust. Working in partnership with the informatics leads for pharmacy and medicine, the palliative care team utilised the capabilities of 'Cerner's' electronic patient prescribing system to develop an 'orderset' of 5 medications, commonly needed at the end of life. The system went live in May 2015 and an early induction session to the new junior doctors was delivered in August 2015.

Aims To evaluate prescribing of anticipatory 'as required' EOL medications, following the introduction of the palliative care 'order-set'.

Methods A retrospective review of anticipatory, 'as required', prescribing for a 1 month period was undertaken 12 months after introducing the prescribing 'order-set'. Criteria applied as for the 2013 National Hospital audit.

Results were compared with local results from 2013. Baseline information from 2013 showed that the Trust under-performed for prescribing for all symptoms: (Agitation- 23\%, Breathlessness- 20\%, Nausea- 14\%, Pain- 27\%, and Secretions7\%).

Twelve months after implementation of the 'order- set' the results were: (Agitation- 68\%, Breathlessness- 74\%, Nausea$68 \%$, Pain- 74\%, and Secretions- 65\%). These results are all above the national average from the 2016 RCP: National End of life care audit.

Conclusions Introduction of an electronic palliative care prescribing 'order-set' for anticipatory EOL medications, has demonstrated considerable improvement in the uptake of anticipatory prescribing for dying patients. This in conjunction with an early palliative care induction education session for junior doctors has demonstrated significant improvements in the quality of prescribing in the end of life phase of life for patients in an acute hospital.

\section{ASSESSING THE MANAGEMENT OF HEART FAILURE PATIENTS IN THE INPATIENT HOSPICE SETTING: A RETROSPECTIVE AUDIT}

Laura Dewhirst, Louise Gilhooley, Kate Hoddell. Severn Hospice

10.1136/bmjspcare-2018-ASPabstracts. 160

Approximately 900,000 people in the UK have heart failure (HF) with $30-40 \%$ dying within a year. The incidence of HF is increasing. Patients have a high symptom burden; patients with HF will have similar numbers of symptoms to cancer patients. As UK life expectancy increases palliative services will need to provide more support to this patient group.

This retrospective audit assessed care from Severn Hospice to HF patients admitted to the hospice since November 2015. NICE guideline 'Chronic Heart Failure in Adults: Management' was used as the gold standard.

Of 962 patients admitted to the hospice, 13 had a diagnosis of HF. 9 had a primary diagnosis and 4 had HF as a comorbidity. The majority of patients were referred for symptom control. At admission patients had a mean of 4.38 (range 17) symptoms. 9 patients had symptoms not in the NICE guideline including constipation, diarrhoea, anxiety, agitation and hallucinations. The majority had more symptoms at admission than at initial referral (mean 4.84). 9 patients had syringe drivers during their admission; 3 were furosemide infusions.

All patients had some advance care planning (ACP). All had a 'do not resuscitate order'. Preferred place of death was documented in 12 patients (1 had no capacity). 9 had a preferred place of care. No patients had an advance directive to refuse treatment. Most ACP was done on admission.

Surprisingly few patients with HF are admitted to the hospice. These patients have a significant symptom burden including some symptoms not always associated with HF. This suggests that the needs of HF patients are not being met. As a result efforts are being made to set up a joint $\mathrm{HF}$ and palliative care MDT. With this and increasing numbers of patients the hospice will continue to gain expertise in managing these patients.

\section{STAFF VIEWS ON CHANGES TO THE MULTI- PROFESSIONAL ELECTRONIC-HANDOVER IN A SPECIALIST PALLIATIVE CARE UNIT: SERVICE DEVELOPMENT PROJECT}

Hayley Evans, Eleanor M Smith, Claire N MacDermott. Sheffield Teaching Hospitals NHS Foundation Trust

\subsection{6/bmjspcare-2018-ASPabstracts. 161}

Background Introducing change to service can be challenging. Following an audit of electronic handover (e-handover), a multi-professional group of palliative care doctors, nurses and allied health professionals, developed a Standard Operational Procedure (SOP) to guide staff and ensure e-handover consistently addressed patients' specific palliative care needs. As part 
of this intervention, multi-professional teaching regarding changes to e-handover was provided, with the intention to engage the whole team in supporting communication within and between professional groups, and to improve patient care. Methods A survey was designed to measure staff views on their perceived impact of the SOP on the handover, communication and patient care. This was administered over a two week period in September 2017 on paper and electronically. Twenty-seven surveys were completed by doctors, nurses and clinical support workers who use e-handover.

Results Ninety-two percent of those surveyed felt that the handover was easier to read, $100 \%$ felt they had a clearer understanding of what information to include on the handover, $92 \%$ had a better understanding of escalation status and $47 \%$ of nurses felt it was easier to explain this status to a doctor out of hours. Assessing advance care planning, 85\% felt they had a better understanding of a patient's preferred place of death. Sixty-six percent of those surveyed felt patient care had improved as a result of these changes. Comments included 'this has been a great development which ensures a more robust and consistent approach to handover. However, $48 \%$ felt the changes had resulted in handover being more time consuming.

Conclusion Multi-professional input to support changes in the e-handover led to improved communication between staff, a clearer understanding of escalation status and preferred place of death for patients, leading to improved patient care. Further work is required to understand the cause of time burdens in completing handover.

\section{A TALE OF TWO SETTINGS: ARE THERE DIFFERENCES IN HOSPICE INPATIENT INITIAL NEEDS \& OUTCOMES DEPENDING ON WHERE THE PATIENT IS ADMITTED FROM?}

FA Malik, S Clarke, D Barclay. St. Wilfrid's Hospice, Eastbourne, East Sussex NHS Healthcare Trust

\subsection{6/bmjspcare-2018-ASPabstracts. 162}

Introduction Understanding patient needs and assessing outcomes are important to improve quality of care. As part of an ongoing evaluation of hospice inpatient (IPU) bed use, the needs \& outcomes of those admitted to the IPU from the local hospital trust and from community settings were compared to examine if there were differences depending on settings admitted from.

Methods Cross-sectional retrospective analysis of consecutive IPU admissions from the local hospital NHS Trust (HA) and admissions from the community (CA) to a specialist palliative 14-bedded hospice from August 16-March 17. Data items collected include patient demographics \& outcome data e.g. IPU admission IPOS, phase of illness (PoI) \& AKPS. All data anonymised. Results analyzed and descriptive statistics utilized.

Results $50 \mathrm{HA}$ and $113 \mathrm{CA}$ admissions during the time period (in addition 24 respite admissions and 6 'out of area' HA not used in analysis). There were no differences in mean age (HA $71.3 \mathrm{yrs}, \mathrm{CA} 71 \mathrm{yrs}$ ) or gender (HA 60\%, CA 53\% male) and no difference in malignant diagnosis between groups (HA $82 \%$, CA $88 \%$ ).

IPU admission mean AKPS was worse in HA (33) than CA (44.6) $(\mathrm{p}<0.005)$ but no difference in mean IPOS (HA 31.2, CA $34, \mathrm{p}=0.1)$.
HA patients were more likely to be in the 'dying phase' (PoI) on IPU admission (14/50,28\%) compared to other phases than CA patients $(9 / 110,8 \%)(\mathrm{p}<0.001)$. Mean IPU length of stay was longer in CA (17.6 days) compared to HA (13.2 days) $(p=0.05)$. HA were more likely to die during that IPU admission compared to CA patients (46/50 (92\%) vs 69/110 $(62 \%)),(p<0.05) .41 / 110$ CA patients were discharged from IPU.

Conclusions Findings highlight potential differences in patient needs and outcomes depending on which setting the patient was admitted from. This will influence development of 24/7 services $\&$ bed utilization. Further analysis is beneficial to clarify findings.

\section{CARE OF PATIENTS WITH PROGRESSIVE SUPRANUCLEAR PALSY IN THE HOSPICE SETTING: AN AUDIT}

Kate Hoddell, Laura Dewhirst, Derek Willis. Severn Hospice

\subsection{6/bmjspcare-2018-ASPabstracts. 163}

Hospices are increasingly focused on improving services for patients with life-limiting non-malignant conditions. Progressive supranuclear palsy (PSP) is a life-limiting progressive neurological disease which can be mistaken for Parkinson's disease early in its course. Symptoms progress rapidly within 5-7 years. Patients with PSP may have a high number of symptoms and will have a poor prognosis.

This retrospective audit aimed to evaluate care provided to patients with PSP referred to Severn Hospice using the guide 'Pathway of Care for PSP: A guide for Health and Social Care Professionals' as the standard. It included all patients under hospice care since 2015.

20 patients were included, 8 of whom continued under hospice care. 7 patients had died and 5 discharged. Time between diagnosis and referral to our service was usually in the first year. Most patients were referred for 'medical assessment and support' and had more than 3 symptoms at referral. 5 patients were admitted to the hospice, 3 of whom died during admission. These patients had at least 4 symptoms at referral.

7 patients had no advance care planning (ACP) completed (defined as a do not resuscitate order (DNAR), preferred place of care (PPC), preferred place of death (PPD) and/or an advance decision to refuse treatment (ADRT)). Of the other patients 13 had a DNAR, 5 had a documented PPC, 5 had a documented PPD and 2 patients had an ADRT. Of the 7 who died, only 2 had a PPD; both achieved their PPD.

This audit showed that while this is a small number of patients, this is a group with a high symptom burden at referral to palliative services. This suggests that palliative medicine has a role early in the patient journey. ACP was not fully completed in any audited patients indicating that this is an area requiring improvement.

\section{IMPROVING ADVANCE CARE PLANNING IN A TERTIARY ONCOLOGY CENTRE}

Harriet Radcliffe, Lori Low, Kiara Parag, Rachel Marshall, Aravind Parkash, Eleanor Smith, Alice Dewdney. Sheffield Teaching Hospitals Foundation Trust

10.1136/bmjspcare-2018-ASPabstracts. 164 